\title{
Assessing maintenance contracts when preventive maintenance is outsourced
}

\author{
Shaomin $\mathrm{Wu}^{1}$ \\ Cranfield University, School of Applied Sciences, Cranfield, \\ Bedfordshire MK43 OAL, United Kingdom
}

\begin{abstract}
In some companies, corrective maintenance is conducted in-house but preventive maintenance might be outsourced. This raises a need to optimise some parameters such as the number of contracts from a perspective of the equipment owner. This paper considers a maintenance policy for such a situation, analyses the roles of the parameters in a PM model, proposes approaches to defining bonus functions, and finally discusses special cases of both the PM policy and the bonus function. Numerical examples are also given to explore the impact of parameters on the expected lifecycle cost rate.

Keywords: maintenance outsourcing, preventive maintenance (PM); corrective maintenance $(\mathrm{CM})$; minimal repair; bonus function.
\end{abstract}

\section{Introduction}

The issues of the optimum strategies of maintenance outsourcing have been studied by a number of authors[1-13]. Existing research has been focused on either outsourcing both preventive maintenance $(\mathrm{PM})$ and corrective maintenance $(\mathrm{CM})$ or outsourcing $\mathrm{CM}$ only. Little attention, however, has been paid to the problem of outsourcing PM, which is discussed in this paper.

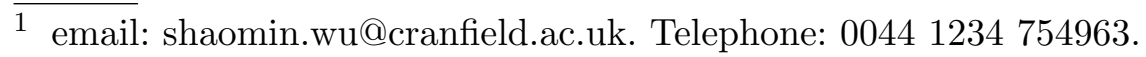




\subsection{Prior work}

In the literature, the types of maintenance outsourcing that have been studied so far include: Type-1 outsourcing: outsourcing both CM and PM[1-9], and Type-2 outsourcing: outsourcing CM[10-13] only.

Type-1 outsourcing: both CM and PM are outsourced. PM policies in the context of outsourcing both PM and CM have been discussed by a number of authors (see [1-6], for example). $[7,8]$ use incentive contracts to induce the contractor to select the maintenance policy that optimises the total profit of the manufacturer and the contractor. In addition to the consideration of CM and PM, [9] considers inspection policies and optimises the contract parameter under different scenarios.

Type-2 outsourcing: Only CM is outsourced. Assuming that a sequence of CM contracts will be made to maintain a piece of equipment and that the service market can provide different kinds of CM contracts to the equipment owner ${ }^{2}$, $[10,11]$ propose methods for determining the optimal series of CM contracts for the equipment's lifetime. [12,13] present decision models for selecting CM contracts based on multi-criteria decision making theory, taking into account different variables such as cost and downtime.

Existing work can be categorised with Table 1.

\section{Here: Table 1}

\subsection{Problems}

In practice, however, there is a possibility that only PM is outsourced but CM is conducted in house. This has been reported from time to time, for example, in [14] where a case about outsourcing PM on power cables is presented.

When outsourcing PM, one might consider the following two options:

Option 1: PM on a piece of equipment will be outsourced to one agent within the lifecyle of the equipment;

$\overline{2}$ In this paper, we call the provider of maintenance service as an agent, and the recipient of the maintenance service as an equipment owner. 
Option 2: PM on a piece of equipment will be outsourced to a number of agents within the lifecyle of the equipment and the quality of PM actions conducted within a contract period can be different.

Remarks on Option 2. Most companies periodically review (common periodicity is 3-5 years) their maintenance procedures, and make contracts with agents within these periods. The maintenance levels can be different from period to period [15]. If we assume that a PM contract starts from a PM action and ends with a PM, there can exist a time interval between two adjacent contracts. Hence, this option will have a time line containing a series events such as (also see Figure 1): a new piece of equipment to start $\rightarrow \tau_{0} \rightarrow T_{1} \rightarrow \tau_{1} \rightarrow$ $T_{2} \rightarrow \tau_{2} \rightarrow \cdots \rightarrow \tau_{N-1} \rightarrow T_{N} \rightarrow \tau_{N} \rightarrow$ replacement, where $\tau_{k}(k=0,1,2, \ldots)$ is the time interval between the end of the $k$-th contract and the start of the $(k+1)$-th contract, and $T_{k}$ represents the length of the $k$ th contract. Within different $T_{k}$, different agents are contracted to undertake PM. Any failures between PM actions are rectified by the equipment owner himself, but a penalty might be incurred on the failures to the agents. The quality of the last PM conducted by an agent in his contract period can be vitally important, as it can affect the remaining life of the equipment being maintained and therefore the optimum choice of maintenance schedules within its subsequent periods. That is, a piece of equipment maintained with good quality of the last PM conducted in a contract period might need fewer PM actions within its remaining lifetime and also fewer failures might occur, whereas a piece of equipment maintained with poor PM quality within a contract period might need more PM actions within its remaining lifetime and more failures might occur. Hence, good quality of the last PM in each contract period can be regarded as a profit to the owner as he will pay less on maintenance within the remaining lifetime of the equipment. In such a case, the owner of the equipment might be willing to pay a bonus to agents for encouraging good PM quality.

As one can see in the following sections, mathematically, Option 1 is a special case of Option 2. Hence, this paper will only discuss Option 2.

\section{Here: Fig 1}

Compared to the first three variables listed in Table 1, Option 2 differs from existing research in the following respects:

Type of outsourcing: Only PM is outsourced; 
Type of penalty: Different penalty schemes can be used.

- The quality of the last PM will be assessed and a bonus might be paid for good quality.

- Penalty for repair not being carried out within specified time limits is not applicable here, as the agent does not undertake any CM upon equipment failures.

Decision variables: The objective is to seek the optimum series of PM contracts with respect to the length $T_{i}$ of a contract, the time interval $\tau_{i}$ between two adjacent contracts, Penalty-2 and bonus schemes.

Hence, if a series of agents are contracted with the equipment owner, it is possible to introduce a new penalty scheme in following:

- failures between PMs can incur Penalty-2 to the agents due to the reliability performance specified in the maintenance contract being violated; and

- a bonus can be offered to the agent if the quality of the last PM in a contract is good.

It should be noted that $[7,8]$ have already used the concept of bonus functions to encourage good maintenance quality in the context of maintenance outsourcing. In [7,8], however, both PM and CM are outsourced, which makes it different from the cases discussed in this paper.

This paper discusses PM policies used in Option 2. The equipment owner outsources PM within fixed periods, in which periodic or sequential PM's might be conducted. He might pay a bonus to agents for their good PM quality. The paper investigates the roles of the parameters in a typical PM model, proposes approaches to defining the bonus functions, derives algorithms to optimise the expected lifecycle cost rate, and discusses special cases of both the PM policy and the bonus function.

The paper considers optimising policies from a perspective of the equipment owner and assumes that costs on CM, PM, and replacement, and the levels of PM are known. This assumption might be rigorous for some cases as some values such as the levels of PM might only be determined by the agents, and unknown to the equipment owner. However, from a perspective of the equipment owner, he needs to assess the optimum values, including contract length, time intervals between adjacent contracts, penalty and bonus, respectively. Hence, such assumptions are necessary.

The rest of the paper is structured as follows. Section 2 presents notation and assumptions. Section 3 derives the lifecycle cost rate, investigates the roles of the parameters in a typical 
PM model, proposes approaches to defining the bonus on PM actions, derives an algorithm to search the optimal solution, and compares two special PM policies. Section 4 presents data examples to look into the validity of the proposed models. Section 5 concludes the findings.

\section{Notation and Assumptions}

\subsection{Notation}

\section{Here: Table 2}

\subsection{Assumptions}

A typical lifecycle of the equipment under study is shown in Fig 1. Suppose the following assumptions hold.

A.1 The planning horizon is infinite.

A.2 The failure intensity function, $h_{0,0}(t)$, of the equipment before any maintenance intervention is conducted is continuous and strictly increasing.

A.3 The lifecycle of the equipment is defined as the time interval between two adjacent replacements. That is, the time in a lifecycle progresses as: a new piece of equipment to start $\rightarrow$ $\tau_{0} \rightarrow T_{1} \rightarrow \tau_{1} \rightarrow T_{2} \rightarrow \tau_{2} \rightarrow \cdots \rightarrow \tau_{N-1} \rightarrow T_{N} \rightarrow \tau_{N} \rightarrow$ replacement. $T_{k}$ is called a the $k$-th contract period. For simplicity, we assume that $T_{k}=T$ in this paper.

A.4 Within the $k$-th contract period, $m_{k}$ PM actions are conducted at time points $(k-1) T+$ $\sum_{i=0}^{k-1} \tau_{i}+t_{k, 1},(k-1) T+\sum_{i=0}^{k-1} \tau_{i}+t_{k, 1}+t_{k, 2}, \ldots,(k-1) T+\sum_{i=0}^{k-1} \tau_{i}+\sum_{i=1}^{m_{k}} t_{k, i}$, respectively, where $k=1, \ldots, N$.

A.5 The quality of the last PM in the $k$-th contract period might be different from the first $\left(m_{k}-1\right)$ th PM's in the same period ${ }^{3}$.

A.6 Repair between PM actions is assumed to be minimal repair. A replacement is carried out at the end of a lifecycle. The equipment used in the replacement is a new, identical

$\overline{3}$ This assumption holds as only the maintenance quality of the last PM in each contract period will affect the reliability of the equipment in its subsequent time periods, and moreover, the case that PM's have the same quality within a contract period is a special case of this assumption. 
item.

A.7 The time on any PM, minimal repair, and replacement are negligible.

\section{Model formulation and analysis}

\subsection{Option 1}

In Option 1, PM is outsourced to one agent.

\subsection{Option 2}

In Option 2, a series of PM contracts are outsourced to different agents. In this case, the equipment owner might be interested in optimising the following parameters:

- time lengths including $T$ and $\tau_{i}$, where $i=0,1,2, \ldots$;

- value of Penalty-2 and bonus value.

Following the above assumptions, we can derive the expected lifecycle cost rate as follows

$$
C\left(N, T, \tau_{0}, \ldots, \tau_{N}, t_{1,1}, \ldots, t_{N, m_{N}}\right)=\frac{G\left(N, T, \tau_{0}, \ldots, \tau_{N}, t_{1,1}, \ldots, t_{N, m_{N}}\right)}{\sum_{k=0}^{N} \tau_{k}+N T},
$$

subject to

$$
\sum_{i=1}^{m_{k}-1} t_{k, i}=T
$$

where

$$
\begin{aligned}
& G\left(N, T, \tau_{0}, \ldots, \tau_{N}, t_{1,1}, \ldots, t_{N, m_{N}}\right) \\
= & c_{o, 1} \int_{0}^{\tau_{0}} h_{0,0}(t) d t+\sum_{k=1}^{N}\left[c_{c, k} \sum_{i=1}^{m_{k}-1} \int_{0}^{t_{k, i}} h_{k, i}(t) d t+c_{o, k} \int_{0}^{\tau_{k}} h_{k, m_{k}}(t) d t+c_{k}\right] \\
& +\sum_{k=1}^{N} Q_{k}\left(h_{k, m_{k}}\left(\tau_{k}\right)\right)+c_{r},
\end{aligned}
$$

$k=1,2, \ldots, N$, and $m_{k}>2$.

In Eq. (1), $\sum_{k=0}^{N} \tau_{k}+N T$ is the expected time length of a lifecycle. In Eq. (3), 
- $c_{o, 1} \int_{0}^{\tau_{0}} h_{0,0}(t) d t$ is the expected cost on failures within time interval $\left(0, \tau_{0}\right)$,

- $c_{c, k} \sum_{i=1}^{m_{k}-1} \int_{0}^{t_{k, i}} h_{k, i}(t) d t$ is the expected cost on failures within time interval $((k-1) T+$ $\left.\sum_{i=0}^{k-1} \tau_{i}+\sum_{j=1}^{i-1} t_{k, j},(k-1) T+\sum_{i=0}^{k-1} \tau_{i}+\sum_{j=1}^{i} t_{k, j}\right)$, where $k \geq 1$

- $c_{o, k} \int_{0}^{\tau_{k}} h_{k, m_{k}}(t) d t$ is the expected cost on failures within time interval $\left(k T+\sum_{i=0}^{k-1} \tau_{i}, k T+\right.$ $\left.\sum_{i=0}^{k-1} \tau_{i}+\tau_{k}\right)$

- $Q_{k}\left(h_{k, m_{k}}\left(\tau_{k}\right)\right)$ is the bonus on the last PM in the $k$-th contract period, and

- $c_{k}$ is the cost paid for the $k$-th contract,

- $c_{r}$ is the replacement cost in a lifecycle.

$Q_{k}\left(h_{k, m_{k}}\left(\tau_{k}\right)\right)$ is the bonus paid to the $k$-th agent who is responsible for the last PM in the $k$-th contract period, which considers PM quality that can affect PM frequency needed in its subsequent contract period(s). This makes Eq. (1) different from the problem of traditional PM policy optimisation (see [16-19], for example), in which the quality of the last PM has not been distinguished. Further discussion on $Q_{k}\left(h_{k, m_{k}}\left(\tau_{k}\right)\right)$ will be carried out in Sections 3.3.

In Eq. (1), the equipment owner needs to optimise $N, t_{k, i}$ and $\tau_{k}$. He might also need to choose the bonus function $Q_{k}\left(h_{k, m_{k}}\left(\tau_{k}\right)\right)$. But before doing so, the bonus function $Q_{k}\left(h_{k, m_{k}}\left(\tau_{k}\right)\right)$ needs to be discussed.

\subsection{The bonus function}

In the section, we discuss the choice of the bonus function $Q_{k}\left(h_{k, m_{k}}\left(\tau_{k}\right)\right)$.

\subsubsection{PM models}

In Eq. (1), defining the function $Q_{k}\left(h_{k, m_{k}}\left(\tau_{k}\right)\right)$ is important. $h_{k, m_{k}}\left(\tau_{k}\right)$ usually defines a PM model. In the reliability literature, PM models are introduced to assess PM quality (see $[20,21]$, or Eq. (4) in the following, for example). The parameters in a PM model play important roles in measuring PM quality. In this section, we analyse the roles of those parameters, then in the following section we define the bonus function $Q_{k}\left(h_{k, m_{k}}\left(\tau_{k}\right)\right)$.

PM models are developed based on adjusting either the failure intensity function of a piece of equipment or its age. After a maintenance action is carried out on a, the failure intensity 
of the equipment is assumed to change from $h_{k, i-1}(t)$ to $h_{k, i}(t)$. If we assume that the quality of the first $\left(m_{k}-1\right)$ th PM actions in the $k$-th contract period are identical and the quality of the last PM is different, a typical case of $h_{k, i}(t)$ associated with $h_{k, i-1}(t)$ given by [20] is

$$
h_{k, i}(t)=a_{k, i} h_{k, i-1}\left(\phi_{k, i} t+\psi_{k, i}\right)+b_{k, i}
$$

where $a_{k, i}(>) 0, \phi_{k, i}(>0), b_{k, i}$ and $\psi_{k, i}$ are parameters.

If we consider Assumption (A.5), we have

- if $1 \leq i \leq m_{k}-1$, then let $a_{k, i}=a_{k, 0}^{\prime}, b_{k, i}=b_{k, 0}^{\prime}, \phi_{k, i}=\phi_{k, 0}^{\prime}$, and $\psi_{k, i}=\psi_{k, 0}^{\prime}$;

- if $i=m_{k}$, then let $a_{k, m_{k}}=a_{k, 1}^{\prime}, b_{k, m_{k}}=b_{k, 1}^{\prime}, \phi_{k, m_{k}}=\phi_{k, 1}^{\prime}$, and $\psi_{k, m_{k}}=\psi_{k, 1}^{\prime}$.

Then, Eq. (4) can also be re-written as

$$
h_{k, i}(t)=A_{k i} h_{0,0}\left(\Phi_{k i} t+\Psi_{k i}\right)+B_{k i}
$$

where $A_{00}=1, \Phi_{00}=1, \Psi_{00}=0, B_{00}=0, m_{0}=0$, for $k \geq 1$,

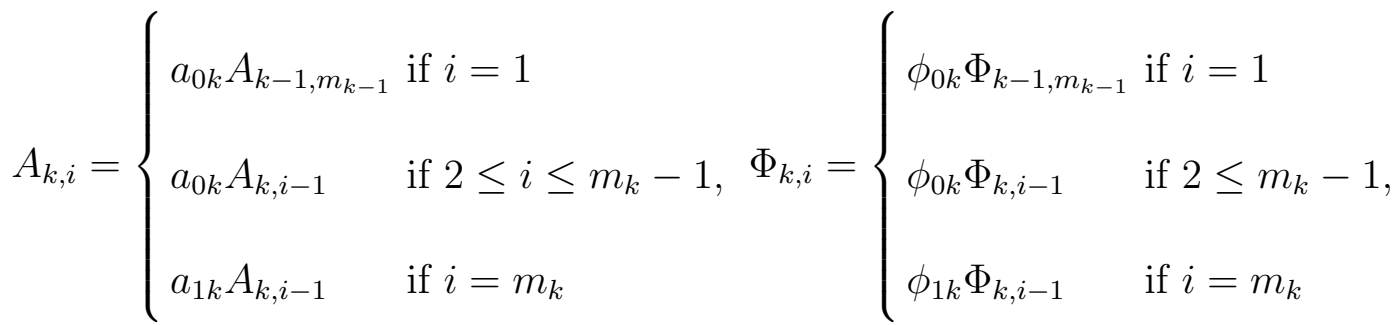

$$
\begin{aligned}
& \Psi_{k, i}=\left\{\begin{array}{ll}
\phi_{0 k} \Psi_{k-1, m_{k-1}}+\psi_{0 k} & \text { if } i=1 \\
\phi_{0 k} \Psi_{k, i-1}+\psi_{0 k} & \text { if } 2 \leq m_{k}-1, \\
\phi_{1 k} \Psi_{k, i-1}+\psi_{1 k} & \text { if } i=m_{k}
\end{array} \text { and } B_{k, i}= \begin{cases}a_{0 k} B_{k-1, m_{k-1}}+b_{0 k} & \text { if } i=1 \\
a_{0 k} B_{k, i-1}+b_{0 k} & \text { if } 2 \leq m_{k}-1 . \\
a_{1 k} B_{k, i-1}+b_{1 k} & \text { if } i=m_{k}\end{cases} \right.
\end{aligned}
$$

The PM model shown in Eq. (4) can be seen as an extension of a wide spectrum of PM models such as those introduced in [21-23], etc.

Estimating the parameters in the bonus function as shown in Eq. (4) is vital. In practise, for example, we might estimate the parameters in Eq. (4) based on data collected from the equipment owner who might have a large number of pieces equipment of the same type maintained and have enough field data, or based on expert elicitation to estimate the parameters. 
A simpler situation might be to count the number of failures within the time period $\tau_{k}$ to assess the quality of the last PM conducted by an agent.

\subsubsection{Some bonus functions}

From Eq. (4), we have

- Assume $h_{k, i-1}\left(\phi_{k, i} t+\psi_{k, i}\right)$ is smaller than 1 . Then we have $\frac{\partial h_{k, i}(t)}{\partial a_{k, i}}=h_{k, i-1}\left(\phi_{k, i} t+\psi_{k, i}\right) \leq$ $\frac{\partial h_{k, i}(t)}{\partial b_{k, i}}=1$, which implies that an improvement in $b_{k, i}$ can affect $h_{k, i-1}\left(\phi_{k, i} t+\psi_{k, i}\right)$ more effectively than $a_{k, i}$ can; and

- As $h_{k, i-1}\left(\phi_{k, i} t+\psi_{k, i}\right)$ is an increasing function, $h_{k, i-1}^{\prime}\left(\phi_{k, i} t+\psi_{k, i}\right)>0$. If $t>1$, then $\frac{\partial h_{k, i}(t)}{\partial \phi_{k, i}}=$ $h_{k, i-1}^{\prime}\left(\phi_{k, i} t+\psi_{k, i}\right) t>\frac{\partial h_{k, i}(t)}{\partial \psi_{k, i}}=h_{k, i-1}^{\prime}\left(\phi_{k, i} t+\psi_{k, i}\right)$, which implies that an improvement in $\phi_{k, i}$ can affect $h_{k, i-1}\left(\phi_{k, i} t+\psi_{k, i}\right)$ more effectively than $\psi_{k, i}$ can.

Remark. The above two bulleted points show that $\phi_{k, i}$ and $b_{k, i}$ in Eq. (4) can play more important roles than parameters $a_{k, i}$ and $\psi_{k, i}$.

From the above discussion, we can define $Q_{k}\left(h_{k, m_{k}}\left(\tau_{k}\right)\right)$, if we assume that it only depends on the parameters, $a_{k, i}, b_{k, i}, \phi_{k, i}$, and $\psi_{k, i}$, in Eq. (4). It might be reasonable to assume that $Q_{k}\left(h_{k, m_{k}}\left(\tau_{k}\right)\right)$ is in inverse proportion to the values $a_{k, i}$ and $\phi_{k, i}$, and in direct proportion to the values $\psi_{k, i}$ and $b_{k, i}$. For example, if PM only changes the parameter $a_{k, i}$ in Eq. (4), then one might estimate the bonus using the following equation.

$$
Q_{k}\left(h_{k, m_{k}}\left(\tau_{k}\right)\right)= \begin{cases}\exp \left(-\theta_{1} a_{k, i}\right) & \text { if } a_{k, i}<1 \\ 0 & \text { otherwise }\end{cases}
$$

where $\theta_{1}$ is a positive parameter.

$a_{k, i}, b_{k, i}, \phi_{k, i}$, and $\psi_{k, i}$ play different roles, according to the above remark, which should be noted when we define/estimate the parameter $\theta_{1}$.

We might also consider the following scenarios on the relationship between $Q_{k}\left(h_{k, m_{k}}\left(\tau_{k}\right)\right)$ and the $m_{k}$ th PM in the $k$-th contract period.

- NoF (number of failures) based approach. $Q_{k}($.$) is a function of \int_{0}^{x} h_{k-1, m_{k-1}}\left(t \mid \tau_{k-1}+T\right) d t-$ $\int_{0}^{x} h_{k, m_{k}}(t) d t: \int_{0}^{x} h_{k, m_{k-1}}\left(t \mid \tau_{k-1}+T\right) d t$ is the number of failures of the equipment within the next $x$ time units, given that the equipment has survived $\tau_{k-1}+T$ time units after the 
last PM in the $(k-1)$-th contract period; $\int_{0}^{x} h_{k, m_{k}}(t) d t$ is the number of failures of the equipment within a given time, $(0, x)$ say, after the last PM in the $k$-th contract period.

$$
\begin{aligned}
& Q_{k}\left(h_{k, m_{k}}\left(\tau_{k}\right)\right) \\
= & \begin{cases}e^{\theta_{2}\left(\int_{0}^{x} h_{k-1, m_{k-1}}\left(t \mid \tau_{k-1}+T\right) d t-\int_{0}^{x} h_{k, m_{k}}(t) d t\right)} & \text { if } \int_{0}^{x} h_{k-1, m_{k-1}}\left(t \mid \tau_{k-1}+T\right) d t>\int_{0}^{x} h_{k, m_{k}}(t) d t \\
0 & \text { otherwise. }\end{cases}
\end{aligned}
$$

where $\theta_{2}$ is a positive parameter.

Using this bonus function, we aim to measure the reduction of the number of failures after the last PM is conducted in the $k$-th contract period.

- Remaining life based approach. $Q_{k}($.$) might also be defined as a function of \int_{0}^{\infty} u d F_{k, m_{k}}(u)-$ $\int_{0}^{\infty} u d F_{k-1, m_{k-1}}\left(u \mid \tau_{k-1}+T\right): \int_{0}^{\infty} u d F_{k, m_{k}}(u)$ is the expected life of the equipment just after the last PM in the $k$-th contract period, and $\int_{0}^{\infty} u d F_{k-1, m_{k-1}}\left(u \mid \tau_{k-1}+T\right)$ is the remaining life of the equipment, given that it has survived for $\tau_{k-1}+T$ time units since the last PM in the $(k-1)$-th contract period. For example, one might define that

$$
\begin{aligned}
& Q_{k}\left(h_{k, m_{k}}\left(\tau_{k}\right)\right) \\
& = \begin{cases}e^{\theta_{3}\left(\int_{0}^{\infty} u d F_{k, m_{k}}(u)-\int_{0}^{\infty} u d F_{k-1, m_{k-1}}\left(u \mid \tau_{k-1}+T\right)\right)} & \text { if } \int_{0}^{\infty} u d F_{k, m_{k}}(u)>\int_{0}^{\infty} u d F_{k-1, m_{k-1}}\left(u \mid \tau_{k-1}+T\right) \\
0 & \text { otherwise, }\end{cases}
\end{aligned}
$$

where $\theta_{3}$ is a positive parameter.

Using this approach will encourage the $(k-1)$-th maintenance company to conduct better maintenance to prolong the time length before the $k$-th company is contracted.

\subsection{Maintenance policies}

We assume that $h_{k, i}(\bullet)$ is defined by Eq. (4), and $Q_{k}\left(h_{k, m_{k}}\left(\tau_{k}\right)\right.$ is defined by Eq. (6). To find the optimal solution that minimises $C\left(N, \tau_{0}, \tau_{1}, \ldots, \tau_{N}, t_{1,1}, \ldots, t_{N, m_{N}}\right)$, we can set the partial derivatives of $C\left(N, \tau_{0}, \tau_{1}, \ldots, \tau_{N}, t_{1,1}, \ldots, t_{N, m_{N}}\right)$ with respect to $\tau_{0}, \tau_{1}, \ldots, \tau_{N}, t_{1,1}, \ldots, t_{N, m_{N}}$ to 0 , respectively, which gives

$$
c_{o, 1} h_{0,0}\left(\tau_{0}\right)\left(\sum_{k=0}^{N} \tau_{k}+N T\right)=G\left(N, T, \tau_{0}, \tau_{1}, \ldots, \tau_{N}, t_{1,1}, \ldots, t_{N, m_{N}}\right)
$$




$$
\begin{gathered}
\left(c_{o, k} A_{k, m_{k}} h_{0,0}\left(\Phi_{k, m_{k}} \tau_{k}+\Psi_{k, m_{k}}\right)+\frac{\partial Q_{k}\left(h_{k, m_{k}}\left(\tau_{k}\right)\right)}{\partial \tau_{k}}\right)\left(\sum_{k=0}^{N} \tau_{k}+N T\right) \\
=G\left(N, T, \tau_{0}, \tau_{1}, \ldots, \tau_{N}, t_{1,1}, \ldots, t_{N, m_{N}}\right)
\end{gathered}
$$

and

$$
\begin{gathered}
c_{c, k} A_{k, i} h_{0,0}\left(\Phi_{k, i} t_{k, i}+\Psi_{k, i}\right)-c_{c, k} A_{k, m_{k}} h_{0,0}\left(\Phi_{k, m_{k}}\left(T-\sum_{i=1}^{m_{k}-1} t_{k, i}\right)\right. \\
\left.+\Psi_{k, m_{k}}\right)+\frac{\partial Q_{k}\left(h_{k, m_{k}}\left(\tau_{k}\right)\right)}{\partial t_{k, i}}=0 .
\end{gathered}
$$

For generality, we have not replaced $\frac{\partial Q_{k}\left(h_{k, m_{k}}\left(\tau_{k}\right)\right)}{\partial t_{k, i}}$ with the one in Eq. (6).

From equations (9) and (11), we have

$$
c_{o, k} A_{k, m_{k}} h_{0,0}\left(\Phi_{k, m_{k}} \tau_{k}+\Psi_{k, m_{k}}\right)+\frac{\partial Q_{k}\left(h_{k, m_{k}}\left(\tau_{k}\right)\right)}{\partial \tau_{k}}=c_{o, 1} h_{0,0}\left(\tau_{0}\right) .
$$

The computing procedure to obtain an optimal schedule can be specified as follows: let $N$ be fixed, then obtain the solutions based on the following steps, after that compare $C\left(N, T, \tau_{0}, \tau_{1}, \ldots, \tau_{N}, t_{1,1}, \ldots, t_{N, m_{N}}\right)$ on the solutions, and then choose those solutions with the minimal

We can specify the computing procedure to obtain an optimal schedule as follows: we can fix $N$, then obtain the solutions based on the following steps, then compare $C\left(N, T, \tau_{0}, \tau_{1}, \ldots, \tau_{N}, t_{1,1}, \ldots, t_{N, m_{N}}\right)$ on the solutions, and then choose those solutions with the minimal $G_{3}\left(N, T, \tau_{0}, \tau_{1}, \ldots, \tau_{N}, t_{1,1}, \ldots, t_{N, m_{N}}\right)$. In the following algorithm, we assume $Q_{k}\left(h_{k, m_{k}}\left(\tau_{k}\right)\right)$ is simply related to $\tau_{k}(k=1,2, \ldots, N)$.

- Let $N=1,2,3, \ldots, n$, where $n$ is a given upper limit;

- Solve Eq. (12), and express $\tau_{k}(k=0, \ldots, N)$ by a function of $\tau_{0}$;

- Solve Eq. (12) with respect to $t_{k, i}$;

- Substitute $\tau_{k}$ and $t_{k, i}$ into Eq. (9) to obtain $\tau_{0}$;

- Substitute $\tau_{0}$ into Eq. (12) to obtain $\tau_{k}$; and

- determine $N$ which minimises Eq. (1).

The above algorithm has simplified the optimisation problem of a complex mixed integer programming to the optimisation problem of an integer programming.

The above algorithm only considers to optimise the frequency of the PM. Similarly, we can 
also optimise the bonus function and penalty costs in each contract periods: he can find the optimum parameters through minimising the expected cost in Eq. (1).

\subsubsection{Special cases of the PM policy}

PM can be conducted either periodically or sequentially. The periodic PM policy is more frequently used due to its simplicity in scheduling, and the sequential PM policy is less frequently used. An interesting PM policy in Option 2 might be a hybrid of the periodic PM policy and the sequential PM policy. In this section, the following two special maintenance policies are investigated (see Figure 1).

- Periodic-sequential (PS) PM policy. Within a given time period T, periodic PM is performed, but the values $\tau_{k}$ are unequal for $k=1,2, \ldots$, ie., $t_{k, 1}=t_{k, 2}=\ldots=t_{k, m_{k}}$ and $\tau_{1} \neq \tau_{2} \neq \ldots, \neq \tau_{N}$. The quality of PM's within a time period $T$ are the same but are different among different time periods $T$ 's. Denote $C_{P S}=C\left(N, T, \tau_{0}, \tau_{1}, \ldots, \tau_{N}, t_{1,1}, \ldots, t_{N, m_{N}}\right)$ as the expected lifecycle cost rate of this case.

- Sequential-periodic (SP) PM policy. Within a given time period T, sequential PM is performed. The values $\tau_{k}$ are equal for $k=1,2, \ldots$, ie, $t_{k, 1} \neq t_{k, 2} \neq \ldots \neq t_{k, m_{k}}$ and $\tau_{1}=\tau_{2}=\ldots,=\tau_{N}$. The quality of PM's within a time period $T$ are the same but are different among different time periods $T$ 's. Denote $C_{S P}=C\left(N, T, \tau_{0}, \tau_{1}, \ldots, \tau_{N}, t_{1,1}, \ldots, t_{N, m_{N}}\right)$ as the expected lifecycle cost rate of this case.

An interesting question is which PM policy, periodic-sequential PM policy or sequentialperiodic PM policy, is better regarding the expected cost rate. The following two Lemmas list sufficient conditions for comparing the two policies.

Lemma 1 Denote $\tau=\frac{1}{N+1} \sum_{k=0}^{N} \tau_{k}$, and assume that $\tau_{0} \geq \tau_{1} \geq \cdots \geq \tau_{k_{0}} \geq \tau \geq \tau_{k_{0}+1} \geq$ $\ldots \tau_{N}$, then

- $\int_{0}^{\tau} h_{0,0}(t) d t+\sum_{k=1}^{N} \int_{0}^{\tau} h_{k, m_{k}}(t) d t \geq \int_{0}^{\tau_{0}} h_{0,0}(t) d t+\sum_{k=1}^{N} \int_{0}^{\tau_{k}} h_{k, m_{k}}(t) d t$ if $\max \left\{h_{0,0}\left(\tau_{0}\right), h_{1, m_{1}}\left(\tau_{1}\right) \ldots, h_{k_{0}, m_{k_{0}}}\left(\tau_{k_{0}}\right)\right\} \leq \min \left\{h_{k_{0}+1, m_{k_{0}+1}}\left(\tau_{k_{0}+1}\right), \ldots, h_{N, m_{N}}\left(\tau_{N}\right)\right\}$.

- $\int_{0}^{\tau} h_{0,0}(t) d t+\sum_{k=1}^{N} \int_{0}^{\tau} h_{k, m_{k}}(t) d t \leq \int_{0}^{\tau_{0}} h_{0,0}(t) d t+\sum_{k=1}^{N} \int_{0}^{\tau_{k}} h_{k, m_{k}}(t) d t$ if $\min \left\{h_{0,0}\left(\tau_{0}\right), h_{1, m_{1}}\left(\tau_{1}\right) \ldots, h_{k_{0}, m_{k_{0}}}\left(\tau_{k_{0}}\right)\right\} \geq \max \left\{h_{k_{0}+1, m_{k_{0}+1}}\left(\tau_{k_{0}+1}\right), \ldots, h_{N, m_{N}}\left(\tau_{N}\right)\right\}$. 
The proofs for the Lemmas in this paper can be found in Appendix.

Lemma 2 Denote $t_{k, 0}=\frac{1}{m_{k}-1} \sum_{i=1}^{m_{k}-1} t_{k, i}, Q_{k}\left(h_{k, m_{k}}\left(\tau_{k}\right)\right)$ is measured with Eq. (6), and assume that $t_{k, 1} \geq t_{k, 2} \geq \cdots \geq t_{k, i_{k}} \geq t_{k, 0} \geq t_{k, i_{k}+1} \geq \ldots t_{k, m_{k-1}}$ for $k=1, \ldots, N$. Then,

- $C_{S P}>C_{S P}$ if $\max \left\{h_{0,0}\left(\tau_{0}\right), h_{1, m_{1}}\left(\tau_{1}\right) \ldots, h_{k_{0}, m_{k_{0}}}\left(\tau_{k_{0}}\right)\right\} \leq \min \left\{h_{k_{0}+1, m_{k_{0}+1}}\left(\tau_{k_{0}+1}\right), \ldots, h_{N, m_{N}}\left(\tau_{N}\right)\right\}$, and

$\min \left\{h_{k, 1}\left(t_{k, 1}\right), \ldots, h_{k, i_{k}}\left(t_{k, i_{k}}\right)\right\} \geq \max \left\{h_{k, i_{k}+1}\left(t_{k, i_{k}+1}\right), \ldots, h_{k, m_{k}}\left(t_{k, m_{k-1}}\right)\right\}$.

- $C_{S P}<C_{S P}$ if $\max \left\{h_{0,0}\left(\tau_{0}\right), h_{1, m_{1}}\left(\tau_{1}\right) \ldots, h_{k_{0}, m_{k_{0}}}\left(\tau_{k_{0}}\right)\right\} \geq \min \left\{h_{k_{0}+1, m_{k_{0}+1}}\left(\tau_{k_{0}+1}\right), \ldots, h_{N, m_{N}}\left(\tau_{N}\right)\right\}$, and $\min \left\{h_{k, 1}\left(t_{k, 1}\right), \ldots, h_{k, i_{k}}\left(t_{k, i_{k}}\right)\right\} \leq \max \left\{h_{k, i_{k}+1}\left(t_{k, i_{k}+1}\right), \ldots, h_{k, m_{k}}\left(t_{k, m_{k-1}}\right)\right\}$.

Lemma 2 gives sufficient conditions for comparing the two PM policies with respect to their expected lifecycle cost rate.

\section{Illustrative examples}

Consider a case where the life distribution of a piece of equipment under consideration is a 2-parameter Weibull distribution at operating state. The failure intensity is assumed to be $h_{0}(t)=\frac{1}{8}\left(\frac{t}{24}\right)^{2}$. That is, its corresponding lifetime distributions is given $F(t)=1-$ $\exp \left\{-\left(\frac{t}{24}\right)^{3}\right\}$. We set $N=3, T=36, c_{r}=5000, c_{o, k}=c_{c, k}=100 e^{0.5 k}, Q_{k}\left(h_{k, m_{k}}\left(\tau_{k}\right)\right)=$ $0.1 e^{\frac{0.8}{a_{k}-1}}$. In Eq. (4), without loss of generality, we set $a_{k, i}=\frac{k+5.2}{k+4}, \phi_{k, i}=1, \psi_{k, i}=0$, and $b_{k, i}=0$.

\subsection{Comparison between the PS and the SP policies}

Using the above parameters, we obtain the optimal PS and SP policies as shown in Tables 3 and 4 , from which one can find that the two policies reach their optimal values when $N=3$. However, the expected lifecycle cost rate of the SP policy is greater than that of the PS policy.

For the optimal policies $N=3$, if we further prolong the length of $\tau$ in the SP policy to let $\tau=\frac{1}{N+1} \sum_{k=0}^{N} \tau_{k}$, we can obtain Table 5 , from which one can find that the expected lifecycle 
cost rate of the SP policy (67.12) is more than 1.4 times as big as that of the PS policy (47.73).

\section{Here: Figure 2.}

\section{Here: Table 3}

\section{Here: Table 4}

\section{Here: Table 5}

\subsection{Impact of PM quality}

Changing parameter $\Delta$ in $a_{k, i}=\frac{k+\Delta}{k+4}$, we can investigate the impact of the parameters $a_{k, i}$ on the expected lifecycle cost rate for the two policies, ie., the PS policy and the SP policy ( as shown in Table 6). For example, the changes of $C_{P S}$ against $\Delta$ are shown in Figure 2, which is a concave curve and the expected long-run cost rate reaches the optimal value when $\Delta=5.2$.

\section{Here: Table 6}

\subsection{Impact of $C M$ cost}

Now, we re-set $c_{o, k}=c_{c, k}=100 e^{c_{m} k}$. That is, we set $N=3, T=36, c_{r}=5000$, $Q_{k}\left(h_{k, m_{k}}\left(\tau_{k}\right)\right)=0.1 e^{\frac{0.8}{a_{k}-1}}$, and $a_{k}=\frac{k+5.2}{k+4}$. We change $c_{m}$ from 0.1 to 1 , the expected lifecycle cost rate shows a linear relationship with $c_{m}$, as shown in Table 7 .

\section{Here: Table 7}

\section{Conclusions}

This paper considers providing equipment owners with approaches to benchmarking various parameters for the case where PM is outsourced. It investigated the roles of the parameters in a PM (PM) model in respect to PM quality, derived an approach to measuring the profit 
of good PM actions, and derived an approach to searching the optimal solutions.

Future research includes:

- The planning horizon is assumed infinite in this paper. Another consideration that can be taken is the scenario of finite planning horizon.

- The contract periods (i.e., $T$ ) in a lifecycle are assumed to be identical in the paper. A reasonable extension can be to assume that they are varying.

\section{Acknowledgements}

I would like to thank Dr Ralf Gitzel for the useful discussion.

\section{References}

[1] J. Jaturonnatee, D. N. P. Murthy, R. Boondiskulchok, Optimal preventive maintenance of leased equipment with corrective minimal repairs, European Journal of Operational Research 174 (1) (2006) 201-215.

[2] J. Pongpech, D. N. P. Murthy, Optimal periodic preventive maintenance policy for leased equipment, Reliability Engineering and System Safety 91 (7) (2006) 772-777.

[3] R. H. Yeh, K. . Kao, W. L. Chang, Optimal preventive maintenance policy for leased equipment using failure rate reduction, Computers and Industrial Engineering 57 (1) (2009) 304-309.

[4] C. Jackson, R. Pascual, Optimal maintenance service contract negotiation with aging equipment, European Journal of Operational Research 189 (2) (2008) 387-398.

[5] W. Chang, H.-C. Lo, Joint determination of lease period and preventive maintenance policy for leased equipment with residual value, Computers and Industrial Engineering 61 (3) (2011) $489-496$.

[6] R. Yeh, K.-C. Kao, W. Chang, Preventive-maintenance policy for leased products under various maintenance costs, Expert Systems with Applications 38 (4) (2011) 3558 - 3562.

[7] H. Tarakci, K. Tang, H. Moskowitz, R. Plante, Incentive maintenance outsourcing contracts for channel coordination and improvement, IIE Transactions (Institute of Industrial Engineers) 38 (8) (2006) 671-684. 
[8] H. Tarakci, K. Tang, H. Moskowitz, R. Plante, Maintenance outsourcing of a multi-process manufacturing system with multiple contractors, IIE Transactions (Institute of Industrial Engineers) 38 (1) (2006) 81-92.

[9] W. Wang, A model for maintenance service contract design, negotiation and optimization, European Journal of Operational Research 201 (1) (2010) 239-246.

[10] A. Lisnianski, I. Frenkel, L. Khvatskin, Y. Ding, Maintenance contract assessment for aging systems, Quality and Reliability Engineering International 24 (5) (2008) 519-531.

[11] Y. Ding, A. Lisnianski, I. Frenkel, L. Khvatskin, Optimal corrective maintenance contract planning for aging multi-state system, Applied Stochastic Models in Business and Industry 25 (5) (2009) 612-631.

[12] A. T. Almeida, Multicriteria modelling of repair contract based on utility and electre i method with dependability and service quality criteria, Annals of Operations Research 138 (1) (2005) $113-126$.

[13] A. De Melo Brito, A. De Almeida Filho, A. De Almeida, Multi-criteria decision model for selecting repair contracts by applying utility theory and variable interdependent parameters, IMA Journal Management Mathematics 21 (4) (2010) 349-361.

[14] S. Alshibani, A. Sharaf, Outsourcing preventive maintenance on underground power transmission cables, in: World Wire and Cable Conference 2007 (JICABLE 2007), Paris, France, 2007.

[15] P. Contri, G. Manna, V. Ranguelova, C. Rieg, M. Bieth, Benchmarking study on implemented organizational schemes, advanced methods and strategies for maintenance optimization, Tech. rep., SENUF - Optimization of Maintenance Programmes at NPPs (2006).

[16] H. Wang, A survey of maintenance policies of deteriorating systems, European Journal of Operational Research 139 (3) (2002) 469-489.

[17] S. Wu, D. Clements-Croome, Optimal maintenance policies under different operational schedules, IEEE Transactions on Reliability 54 (2) (2005) 338-346.

[18] S. Wu, D. Clements-Croome, Preventive maintenance models with random maintenance quality, Reliability Engineering and System Safety 90 (1) (2005) 99-105.

[19] W. Wang, D. Banjevic, M. Pecht, A multi-component and multi-failure mode inspection model based on the delay time concept, Reliability Engineering and System Safety 95 (2010) 912-920.

[20] S. Wu, M. J. Zuo, Linear and nonlinear preventive maintenance models, IEEE Transactions on Reliability 59 (1) (2010) 242-249. 
[21] M. Kijima, H. Morimura, Y. Suzuki, Periodical replacement problem without assuming minimal repair, European Journal of Operational Research 37 (2) (1988) 194-203.

[22] T. Nakagawa, Sequential imperfect preventive maintenance policies, IEEE Transactions on Reliability 37 (3) (1988) 295-298.

[23] M. Kijima, Some results for repairable systems with general repair, Journal of Applied Probability 26 (1) (1989) 89-102. 


\section{Appendix}

\section{Proof of Lemma 1}

$$
\begin{aligned}
& \int_{0}^{\tau} h_{0,0}(t) d t+\sum_{k=1}^{N} \int_{0}^{\tau} h_{k, m_{k}}(t) d t-\int_{0}^{\tau_{0}} h_{0,0}(t) d t-\sum_{k=1}^{N} \int_{0}^{\tau_{k}} h_{k, m_{k}}(t) d t \\
& =\int_{\tau_{0}}^{\tau} h_{0,0}(t) d t-\sum_{k=1}^{k_{0}} \int_{\tau}^{\tau_{k}} h_{k, m_{k}}(t) d t+\sum_{k=k_{0}+1}^{N} \int_{\tau_{k}}^{\tau} h_{k, m_{k}}(t) d t \\
& \geq-\max \left\{h_{0,0}\left(\tau_{0}\right), h_{1, m_{1}}\left(\tau_{1}\right) \ldots, h_{k_{0}, m_{k_{0}}}\left(\tau_{k_{0}}\right)\right\} \sum_{k=0}^{k_{0}}\left(\tau_{k}-\tau\right) \\
& +\min \left\{h_{k_{0}+1, m_{k_{0}+1}}\left(\tau_{k_{0}+1}\right), \ldots, h_{N, m_{N}}\left(\tau_{N}\right)\right\} \sum_{k=k_{0}+1}^{N}\left(\tau-\tau_{k}\right) \\
& \geq-\min \left\{h_{k_{0}+1, m_{k_{0}+1}}\left(\tau_{k_{0}+1}\right), \ldots, h_{N, m_{N}}\left(\tau_{N}\right)\right\} \sum_{k=0}^{k_{0}}\left(\tau_{k}-\tau\right) \\
& +\min \left\{h_{k_{0}+1, m_{k_{0}+1}}\left(\tau_{k_{0}+1}\right), \ldots, h_{N, m_{N}}\left(\tau_{N}\right)\right\} \sum_{k=k_{0}+1}^{N}\left(\tau-\tau_{k}\right) \\
& =\min \left\{h_{k_{0}+1, m_{k_{0}+1}}\left(\tau_{k_{0}+1}\right), \ldots, h_{N, m_{N}}\left(\tau_{N}\right)\right\} \sum_{k=0}^{N}\left(\tau-\tau_{k}\right) \geq 0
\end{aligned}
$$

This proves the 1st bulleted item in Lemma 1.

Similarly, we can prove the 2nd bulleted item in Lemma 1. 


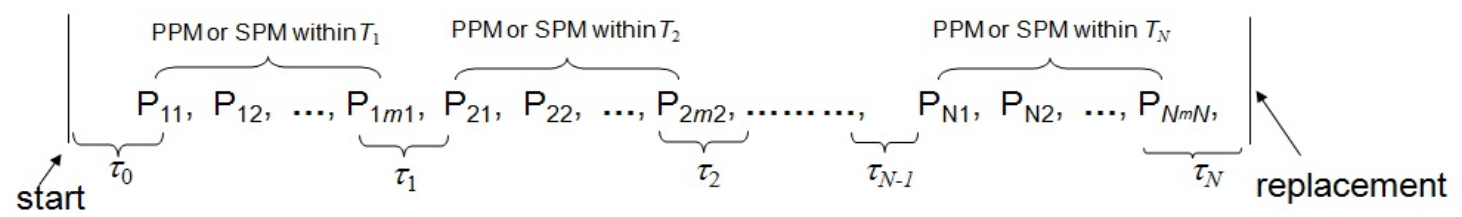

Fig. 1. A PM model $\left(T_{k}=\right.$ the length of the $k$-th contract, PPM=periodic PM, SPM=Sequential $\mathrm{PM}, \mathrm{P}_{k i}=$ the $i$-th PM action in the $k$-th contract).

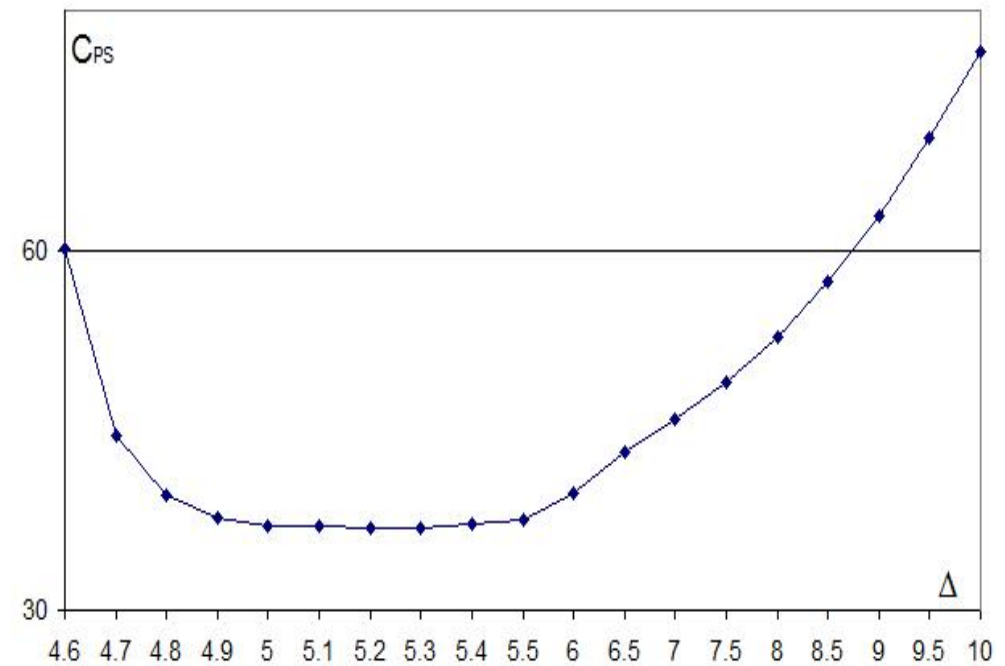

Fig. 2. Impact of PM quality on the expected long-run cost rate. 
Table 1

A summary of some existing papers.

\begin{tabular}{llll} 
Type of outsourcing & Type of penalty $^{\text {a }}$ & Decision variables & References \\
\hline PM \& CM & Penalty-1 \& Penalty-2 & PM policy & {$[1-3]$} \\
PM \& CM & Penalty-1 or Penalty-2 & PM policy or else & {$[4-6,9]$} \\
CM & Penalty-1 & CM contracts & {$[10-13]$} \\
\hline
\end{tabular}

${ }^{\mathrm{a}}$ Penalty-1 : Penalty for repair not being carried out within specified time limits; Penalty-2: Penalty for equipment failures. 
Table 2

Notation.

$N$ number of contracts before a replacement is carried out;

$k$ number of the contracts that the equipment has been passed, $k=0,1,2, \ldots, N$;

$i$ number of the PM actions that an agent has carried out;

$m_{i} \quad$ number of PM actions performed by the $i$-th contractor;

$\tau_{k}$ time interval between the end of the $(k)$-th contract period;

and the start of the $(k+1)$-th contract period;

$t_{k, i} \quad$ time interval between the $i$-th and the $(i+1)$-th PM in the $k$-th contract;

$T$ length of a contract period;

$F_{k, i}(t)$ failure distribution function after the $i$ th PM in the $k$-th contract period;

$h_{k, i}(t)$ failure intensity function after the $i$ th PM in the $k$-th contract period;

$m_{k} \quad$ number of PM's within the $k$-th contract period;

$c_{c, k} \quad$ cost per CM within the $k$-th period after the penalty cost due to the failure has been paid. $c_{c, k}$ can be negative or positive;

$c_{o, k} \quad$ cost per CM during the period of the $\tau_{k}$ after the penalty cost due to the failure has been paid. $c_{o, k}$ can be negative or positive;

$c_{k} \quad$ value of the $k$-th contract;

$c_{r} \quad$ cost per replacement;

$Q_{k}($.$) bonus function.$ 
Table 3

The expected lifecycle cost rate of the PS policy.

\begin{tabular}{|c|c|c|c|c|c|c|c|c|c|c|c|c|c|c|}
\hline$N$ & $\tau_{1}$ & $m_{1}$ & $\tau_{2}$ & $m_{2}$ & $\tau_{3}$ & $m_{3}$ & $\tau_{4}$ & $m_{4}$ & $\tau_{5}$ & $m_{5}$ & $\tau_{6}$ & $m_{6}$ & $\tau_{7}$ & $C_{P S}$ \\
\hline 1 & 34.13 & 3 & 12.26 & & & & & & & & & & & 75.98 \\
\hline 2 & 28.71 & 3 & 11.99 & 10 & 2.78 & & & & & & & & & 53.75 \\
\hline 3 & 27.06 & 2 & 12.59 & 4 & 5.04 & 15 & 1.00 & & & & & & & 47.73 \\
\hline 4 & 30.04 & 1 & 15.57 & 3 & 6.83 & 5 & 2.65 & 18 & 1.01 & & & & & 58.85 \\
\hline 5 & 43.59 & 3 & 18.21 & 4 & 8.76 & 5 & 3.98 & 6 & 1.51 & 20 & 1.15 & & & 123.89 \\
\hline 6 & 63.79 & 2 & 29.68 & 2 & 14.27 & 3 & 6.49 & 3 & 3.04 & 6 & 1.55 & 20 & 1.27 & 265.34 \\
\hline
\end{tabular}

Table 4

The expected lifecycle cost of the SP policy.

\begin{tabular}{c|ccccccccccccc|c}
\hline$N$ & $\tau_{1}$ & $m_{1}$ & $\tau_{2}$ & $m_{2}$ & $\tau_{3}$ & $m_{3}$ & $\tau_{4}$ & $m_{4}$ & $\tau_{5}$ & $m_{5}$ & $\tau_{6}$ & $m_{6}$ & $\tau_{7}$ & $C_{P S}$ \\
\hline 1 & 21.31 & 2 & 21.31 & & & & & & & & & & & 83.17 \\
\hline 2 & 8.64 & 2 & 8.64 & 5 & 8.64 & & & & & & & & & 65.15 \\
\hline 3 & 3.85 & 2 & 3.85 & 4 & 3.85 & 6 & 3.85 & & & & & & & 62.34 \\
\hline 4 & 2.32 & 2 & 2.32 & 2 & 2.32 & 4 & 2.32 & 7 & 2.32 & & & & & 83.62 \\
\hline 5 & 1.13 & 2 & 1.13 & 3 & 1.13 & 4 & 1.13 & 6 & 1.13 & 18 & 1.13 & & & 235.17 \\
\hline 6 & 1.10 & 2 & 1.10 & 3 & 1.10 & 3 & 1.10 & 5 & 1.10 & 5 & 1.10 & 24 & 1.10 & 358.79 \\
\hline
\end{tabular}

Table 5

Comparison between the PS and the SP policies.

\begin{tabular}{|c|c|c|c|c|c|c|c|c|c|c|c|c|c|c|c|c|c|}
\hline Policies & $\tau, \tau_{1}$ & \multicolumn{2}{|c|}{$t_{1}, t_{1 i}$} & $\tau, \tau_{2}$ & \multicolumn{4}{|c|}{$t_{2}, t_{2 i}$} & $\tau, \tau_{3}$ & \multicolumn{6}{|c|}{$t_{3}, t_{3 i}$} & $\tau, \tau_{3}$ & $C_{P S}, C_{S P}$ \\
\hline PS & 32 & 18 & 18 & 20 & 9 & 9 & 9 & 9 & 11 & 6 & 6 & 6 & 6 & 6 & 6 & 6 & 47.73 \\
\hline SP & 11 & 19 & 17 & 11 & 10 & 9 & 9 & 8 & 11 & 7 & 7 & 6 & 6 & 5 & 5 & 11 & 62.34 \\
\hline SP & 17.25 & 19 & 17 & 17.25 & 10 & 9 & 9 & 8 & 17.25 & 8 & 6 & 6 & 6 & 5 & 5 & 17.25 & 67.12 \\
\hline
\end{tabular}

Table 6

Impact of PM quality on the expected lifecycle cost rate.

\begin{tabular}{|c|cccccccccc|}
\hline$\Delta$ & 4.6 & 4.7 & 4.8 & 4.9 & 5.0 & 5.1 & 5.2 & 5.3 & 5.4 & 5.5 \\
\hline$C_{P S}$ & 60.13 & 44.56 & 39.53 & 37.72 & 37.02 & 37.02 & 36.77 & 36.92 & 37.16 & 37.48 \\
\hline$C_{S P}$ & 325.59 & 82.58 & 69.45 & 66.74 & 63.64 & 63.89 & 64.15 & 66.30 & 66.91 & 68.88 \\
\hline$\Delta$ & 6 & 6.5 & 7 & 7.5 & 8 & 8.5 & 9 & 9.5 & 10 & \\
\hline$C_{P S}$ & 39.81 & 43.22 & 46.00 & 49.06 & 52.78 & 57.41 & 62.84 & 69.42 & 76.62 & \\
\hline$C_{S P}$ & 87.32 & 110.15 & 143.54 & 187.78 & 246.64 & 314.86 & 416.21 & 481.74 & 578.23 & \\
\hline
\end{tabular}


Table 7

Impact of CM cost on the expected lifecycle cost rate.

\begin{tabular}{|c|cccccccccc|}
\hline$c_{c, k}$ & 0.1 & 0.2 & 0.3 & 0.4 & 0.5 & 0.6 & 0.7 & 0.8 & 0.9 & 1 \\
\hline$C_{P S}$ & 31.34 & 32.67 & 34.03 & 35.42 & 36.77 & 38.17 & 39.68 & 41.32 & 43.15 & 45.25 \\
\hline
\end{tabular}

